\title{
Personalized dynamic phantom of the right and left ventricles based on patient-specific anatomy for echocardiography studies - preliminary results
}

\author{
João Gomes-Fonseca ${ }^{1,2}$, Pedro Morais ${ }^{1,2,3,4}$, Sandro Queirós ${ }^{1,2,4,5}$, Fernando Veloso ${ }^{1,2,6}$, António C.M. Pinho ${ }^{6}$, Jaime C. \\ Fonseca $^{5}$, Jorge Correia-Pinto ${ }^{1,2,7}$, João L. Vilaça ${ }^{8}$ \\ ${ }^{1}$ Life and Health Sciences Research Institute (ICVS), School of Medicine, University of Minho, Braga, Portugal \\ ${ }^{2}$ ICVS/3B's - PT Government Associate Laboratory, Braga/Guimarães, Portugal \\ ${ }^{3}$ Instituto de Ciência e Inovação em Engenharia Mecânica e Engenharia Industrial, Faculdade de Engenharia, Universidade do \\ Porto, Portugal \\ ${ }^{4}$ Lab on Cardiovascular Imaging and Dynamics, Department of Cardiovascular Sciences, KULeuven - University of Leuven, \\ Leuven, Belgium \\ ${ }^{5}$ Algoritmi Center, School of Engineering, University of Minho, Guimarães, Portugal \\ ${ }^{6}$ Department of Mechanical Engineering, School of Engineering, University of Minho, Guimarães, Portugal \\ ${ }^{7}$ Department of Pediatric Surgery, Hospital of Braga, Braga, Portugal \\ ${ }^{8} 2 \mathrm{Ai}$ - Polytechnic Institute of Cávado and Ave, Barcelos, Portugal
}

\begin{abstract}
Dynamic phantoms of the heart are becoming a reality, with their use spread across both medical and research fields. Their purpose is to mimic the cardiac anatomy, as well as its motion. This work aims to create a dynamic, ultrasoundcompatible, realistic and flexible phantom of the left and right ventricles, with application in the diagnosis, planning, treatment and training in the cardiovascular field for studies using echocardiography. Here, we focus on its design and production with polyvinyl alcohol cryogel (PVA-C), to be assembled with a pump and an electromechanical (E/M) system in a water tank. Based on a patient-specific anatomical model and produced using a 3D printing technique and molding, the PVA-C phantom mimics the ventricles' natural anatomy and material properties, while the pump and $\mathrm{E} / \mathrm{M}$ systems mimic the natural movements and pressures. The PVA-C phantom was assessed by imaging and measuring it using a four-dimensional ultrasound machine. The PVA-C phantom demonstrated to be a versatile option to produce patient-specific biventricular models, preserving their shape after manufacturing and presenting good echogenic properties. Both chambers were clearly seen in the ultrasound images, together with the interventricular septum and the myocardial wall. Automated left ventricle measures revealed a decrease of its volume with regard to the designed model $(98 \mathrm{ml}$ to $74 \mathrm{ml}$ ). Overall, the preliminary results are satisfactory and encourage its use for the abovementioned purposes.
\end{abstract}

Keywords - patient-specific; phantom; PVA-C; manufacturing; echocardiography;

This work has been funded by FEDER funds through the Competitiveness Factors Operational Programme (COMPETE), and by National funds through the Foundation for Science and Technology (FCT) under the project POCI-01-0145FEDER-007038 and EXPL/BBB-BMD/2473/2013, and by the projects NORTE01-0145-FEDER-000013 and NORTE-01-0145-FEDER-024300, supported by the NORTE 2020, under the Portugal 2020 Partnership Agreement, through the European Regional Development Fund (FEDER). J. Gomes-Fonseca, P. Morais, S. Queirós, and F. Veloso were funded by FCT under the Ph.D. grants PD/BDE/113597/2015, SFRH/BD/95438/2013, SFRH/BD/93443/2013, and $\mathrm{SFRH} / \mathrm{BD} / 131545 / 2017$, respectively.

\section{INTRODUCTION}

D hantom (or imaging phantom) is an object designed to be imaged by a medical imaging equipment. It is used to study, assess and tune the parameters of the imaging equipment and/or software. Phantoms must have similar characteristics to the tissues and organs and be built to respond according to the technical specificities of the equipment.

Given the importance of imaging in the cardiovascular field, heart phantoms can be used to improve diagnosis, planning, treatment and training [1]-[4]. Because of the dynamic nature of the cardiac muscle, a heart phantom should be able to mimic both the anatomical and physiological changes that occur throughout the cardiac cycle.

The aim of this work was to develop a dynamic heart phantom for echocardiography studies using real anatomy data of the left and right ventricles ( $\mathrm{LV}$ and $\mathrm{RV}$, respectively). The development of this phantom is integrated in a research project that intends to develop a device to help in diagnosis, planning, treatment, and training in the cardiovascular field. Thereby, to answer this goal, this device must be compatible with ultrasound imaging, flexible, adaptable in terms of simulated heart rate and pressure, and able to be inserted with catheters and other small instruments (for the training of surgical procedures). This work focuses mainly on the design/production of the biventricular phantom.

\section{STATE-OF-THE-ART}

In the literature, some scientific publications and commercial systems have already been presented. Each one presents different concepts and applications for dynamic heart phantoms in echocardiography.

Olszewski et al. developed an echocardiographic model of the left ventricle (LV) to validate the numerical model of the 
myocardial function [1]. The phantom had a shape of a tube with internal diameter of $5 \mathrm{~cm}$, wall thickness of $1 \mathrm{~cm}$ and length of $12 \mathrm{~cm}$. Polyvinyl alcohol (PVA) was used to generate an elastic model and to mimic the mechanical and acoustical properties of the real tissues. Then, a commercial available computed-controlled dynamic piston pump (Vivitro Labs, Victoria, British Columbia, Canada) was used to pump water into the model and consequently deform it. This pump system is widely used to simulate cardiac flow, where the piston motion is easily controlled by a preconfigured waveform, e.g. an electrocardiography (ECG) signal.

Plewinska et al. developed a two chambers phantom model to study the mechanical properties of the myocardium [2]. The system relies on a biventricular model, i.e. LV and RV, which was constructed through a mold made of casting polyurethane. The mold had the shape of a truncated thick-walled ellipsoid with an inner partition (interventricular wall). A uniform myocardial wall thickness was used along the phantom. In order to generate the elastic model, a PVA aqueous solution was also used. Moreover, the phantom model was kept in a water tank coated with ultrasound-absorber material to improve the ultrasound image. A hydraulic system was used to deform the phantom model and simulate the heart motion. As such, two reciprocally moving pistons cyclically deform (inflate and deflate) the cardiac model. These pistons were connected to a step motor to electronically define the cardiac flow rhythm.

Heyde et al. modified this last phantom model in order to validate a novel strategy to estimate three-dimensional (3D) regional cardiac motion and strain in ultrasound imaging [3]. Instead of the biventricular mold, the authors used a thickwalled univentricular phantom with realistic LV dimensions and intracavity volume. Moreover, a glycerin solution was combined with PVA and water to generate the phantom material, resulting in a scattered myocardial wall. These scatters resulted in speckle patterns upon the ultrasound acquisition, consequently creating a more realistic image.

Similarly, Cygan et al. used the abovementioned univentricular model for cardiac motion assessment in ultrasound and magnetic resonance imaging (MRI) [4]. Instead of a step motor combined with cyclic pistons, the authors applied the commercially available pump system (Vivitro Labs, Victoria, British Columbia, Canada). The ultrasound image acquisition was performed without any special attention, while during the MRI acquisition, the pump system was kept outside of the room.

Regarding the commercial available solutions, two different setups are available, namely: a beating heart phantom (The Chamberlain Group, Great Barrington, Massachusetts, United States) [5], [6] and a dynamic multi-modal heart phantom (Shelley Medical Imaging Technologies, London, Ontario, Canada) [7]. The former uses an air pump attached to the cardiac structure to inflate/deflate the LV chamber, creating a realistic cardiac contraction. The pump system is controlled through an external microcontroller, where a simulated real-time ECG trigger is used to define the heart rate. In the second system, the phantom includes an accurate and realistic representation of the $L V$ and $R V$. Two servomotors controlled by an external software are used to define the torsional heart motions, heart stretching and compress motion. In order to acquire realistic ultrasound images of the cardiac muscle, PVA hydrogel doped with iodine and graphite was used to mimic the myocardial tissue.

Dynamic phantoms to simulate the aortic and mitral valves' motion have also been presented. In [8], a phantom with functional mitral and aortic valves was developed, which can be used to simulate valve repair/replacement procedures with the use of fluoroscopy, ultrasound or MRI-based guidance. The phantom consists of a LV, an atrial reservoir capable of holding the left and right atria, a valve sheet containing both valves, and a microcontroller-based pneumatic actuator system. The phantom model was constructed using silicone for the LV, a separate silicone assembly for the mitral and aortic valves, and a box for the left atrium. Regarding the dynamic system, a total of six pneumatic cylinders controlled by a microcontroller were used to define the three contractile loops, namely the LV contraction, and both mitral and aortic valves' motion.

Furthermore, atrial models of the heart have been also presented to create realistic environments to potentiate the study of different pathologies and minimally-invasive procedures. In [9], a physical phantom of the left atrium and pulmonary veins was constructed from a patient dataset. Using a rapid prototyping printer, a surface model was generated and 3D-printed. The authors studied and quantified different registration strategies for left atrial cardiac ablation procedures. Laing et al. generated a patient-specific atrial phantom from geometric models based on CT images [10]. The geometric models were converted into physical representations and used to build a mold. The atria were then molded using tissue-mimicking materials, imaged using CT, and compared to the original data. Morais et al. also presented a patient-specific atrial model for planning and training of inter-atrial septal wall interventions [11]. In this work, two
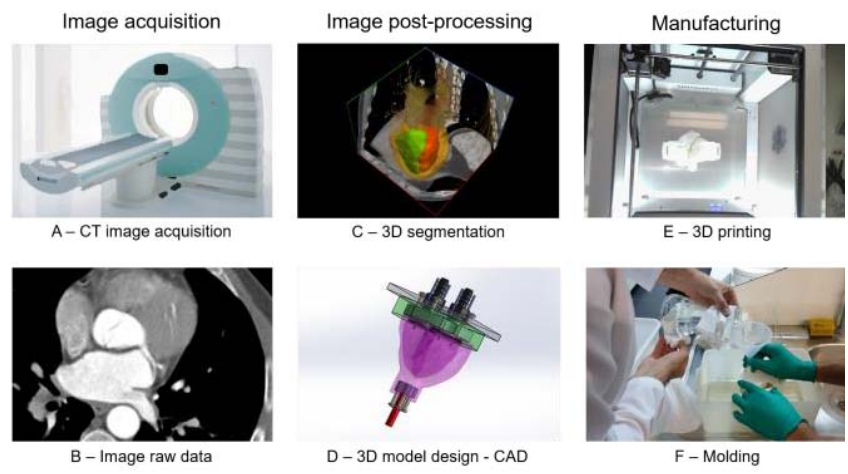

Fig. 1 - The process chain used to produce the phantom consists of three main steps: the image acquisition, the image post-processing and the model manufacturing. A - Images were acquired using a computer tomography (CT) scan. B - The image raw data are then transferred to a computer unit for post-processing. $\mathrm{C}$ - At this time, a three-dimensional (3D) segmentation is performed and $-\mathrm{D}-\mathrm{a}$ computer-aided design (CAD) model of the segmented structures is designed, with its respective molds. E - These molds were manufactured using a 3D printer. F - The flexible and personalized phantom was molded by pouring material into the molds 

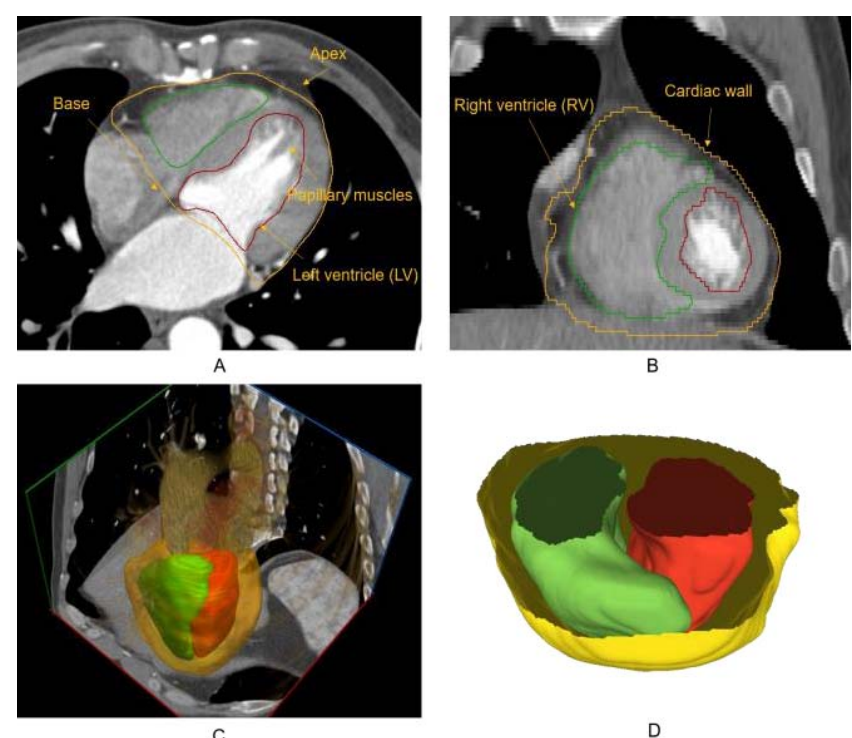

D

Fig. 2 - Segmentation of the left ventricle (LV), right ventricle (RV) and cardiac wall. A - Long axis view. B - Short axis view. C - Final threedimensional segmentation. D - Final smoothed surfaces, with cardiac valves' leftovers removed.

different flexible materials (silicone and polyvinyl alcohol cryogel) were compared for phantom's construction.

\section{METHODOLOGY}

Using image segmentation, computer-aided design (CAD) and rapid prototyping, a patient-specific biventricular phantom model was produced. This phantom was designed to be submerged in a water tank (enabling ultrasound acquisitions) and to be connected to a pump (that mimics the heart inflation/deflation) and an electromechanical (E/M) system (mimicking the heart torsion). Fig. 1 summarizes the process chain involved to manufacture the proposed phantom.

\section{A. Image acquisition}

The biventricular phantom was created from a fourdimensional (4D) cardiac computed tomography (CT) dataset. A DICOM sample dataset from OsiriX, named MAGIX, was used [12]. This dataset is composed by ten sequential moments of the cardiac cycle, each with 76 images with a slice thickness of $3 \mathrm{~mm}$, a matrix of $512 \times 512$ and a pixel spacing of $0.402 \times 0.402 \mathrm{~mm}$. The CT images were acquired using the SOMATOM Sensation 64 (Siemens Medical Solutions, Forchheim, Germany). The X-ray tube current and peak voltage were set to $550 \mathrm{~mA}$ and $120 \mathrm{kV}$, respectively.

\section{B. $3 D$ segmentation and surface processing}

The heart was segmented in the systolic phase, given that it represents the time point where the ventricles have a smaller volume. The diastolic phase (i.e. the maximum volume) will be simulated through the pump (by ejecting water inside the phantom model) and the E/M systems.
Initially, both ventricles' chambers and myocardium were manually segmented in several 2D slices of the CT image using an interactive segmentation framework, The Medical Imaging Interaction Toolkit - MITK (German Cancer Research Center, Heidelberg, Germany) [13]. Due to the presence of CT intravenous contrast, the LV chamber was easily delineated. The valve region and papillary muscles were included on the LV contour, obtaining a uniform and regular shape of the left ventricle. Since no contrast is present within the RV, its segmentation was harder to perform. The RV was segmented by preserving its uniform shape and the septum anatomy (separating LV and RV). The segmentation of the ventricular chambers and wall was performed from the cardiac apex to the ventricles' base (Fig. 2-A/B).

The final surfaces were obtained using a 3D interpolation technique based on radial basis functions, followed by manual corrections if deemed necessary (Fig. 2-C) [14].

Then, the abovementioned surfaces were imported in the stereolithographic file format into the MeshLab software (Visual Computing Lab, ISTI - CNR, Italy) and were then post-processed. Hereto, the surfaces were smoothed and the leftovers of the cardiac valves removed (Fig. 2-D).

\section{C. $3 D$ model design}

As described in [11], we used the Geomagic software (3D Systems, Rock Hill, South Carolina, United States) to convert the previous surfaces (triangular meshes) into solid objects. These solids were exported in step file format into a CAD software (SolidWorks, Dassault Systèmes SolidWorks Corporation, Waltham, Massachusetts, United States).

The first step was to create the ventricular chambers. Through the "cavity" command in SolidWorks, the chambers were removed from the cardiac wall solid using the internal molds of the LV and RV. As aforementioned, the phantom will be integrated in a water tank with a pump and an E/M system (Fig. 3). The pump will be used to increase/decrease the pressure and volume in the phantom cavities through the movement of the piston available on the pump, simulating the different cardiac phases. The diastolic phase will be obtained when the maximum volume is reached, while the systolic phase when the pressure is reduced and the phantom model recovers its initial volume. The E/M system will mimic the

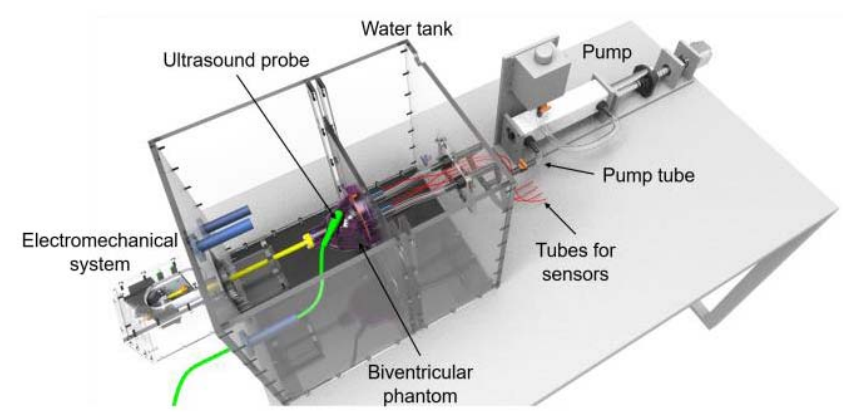

Figure 3 - Complete setup: biventricular phantom, water tank, pump and electromechanical system. 


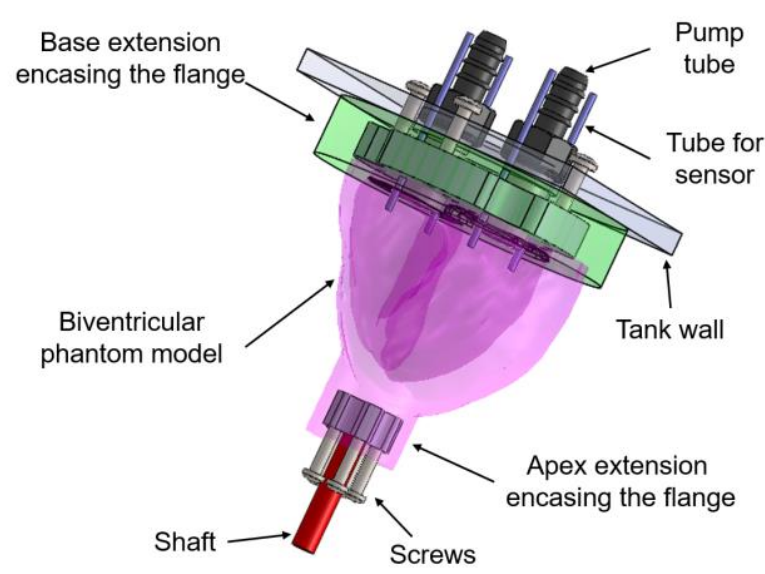

Fig. 4 - Biventricular phantom model with added features.

translational/torsional movements of the heart. Due to these details, some features were added to the model (Fig. 4):

- the apex has a flange to achieve translational and torsional movements (applied by the E/M system);

- the base has a flange to be fixed into the wall tank;

- the base presents two holes to connect the chambers to the pump and four smaller holes to sense the chambers internally;

- the complete model will be produced in two parts. The biventricular model with the apex extension is produced first and then the base extension. The purpose is to remove the RV and $\mathrm{LV}$ internal molds without damaging the phantom model.

In detail, the following parts were designed to produce the biventricular phantom model with the apex extension (Fig. 5A): a cardiac external mold; a RV internal mold; a LV internal mold; a support for the internal molds; and an apex flange. To produce the base extension (Fig. 5-B): an external mold and a flange were designed. The tubes and screws were designed based on standardized measures and joined to the final model (Fig. 5-C).

\section{D. $3 D$ model production}

At this stage, the designed molds were converted to gcode file format using the Cura software (Ultimaker B.V., Geldermalsen, The Netherlands) and then 3D printed in polylactic acid material using an Ultimaker II (Fig. $1-$ E). This device uses the fused deposition modelling technique to print objects. The following machine parameters were used:

- Speed: $60 \%$;

- Nozzle temperature: $225^{\circ} \mathrm{C}$;

- Build-plate temperature: $60^{\circ} \mathrm{C}$;

- Material Flow: $100 \%$;

- Fan Speed: $50 \%$.

The flanges, made of acrylic with $5 \mathrm{~mm}$ of thickness, were cut using a laser cutting machine (GN640; Gbos Laser Technology Company, Dongguan City, Guangdong Province, China).

After the molds' construction, the phantom was formed out of a polyvinyl alcohol cryogel (PVA-C). PVA-C is produced
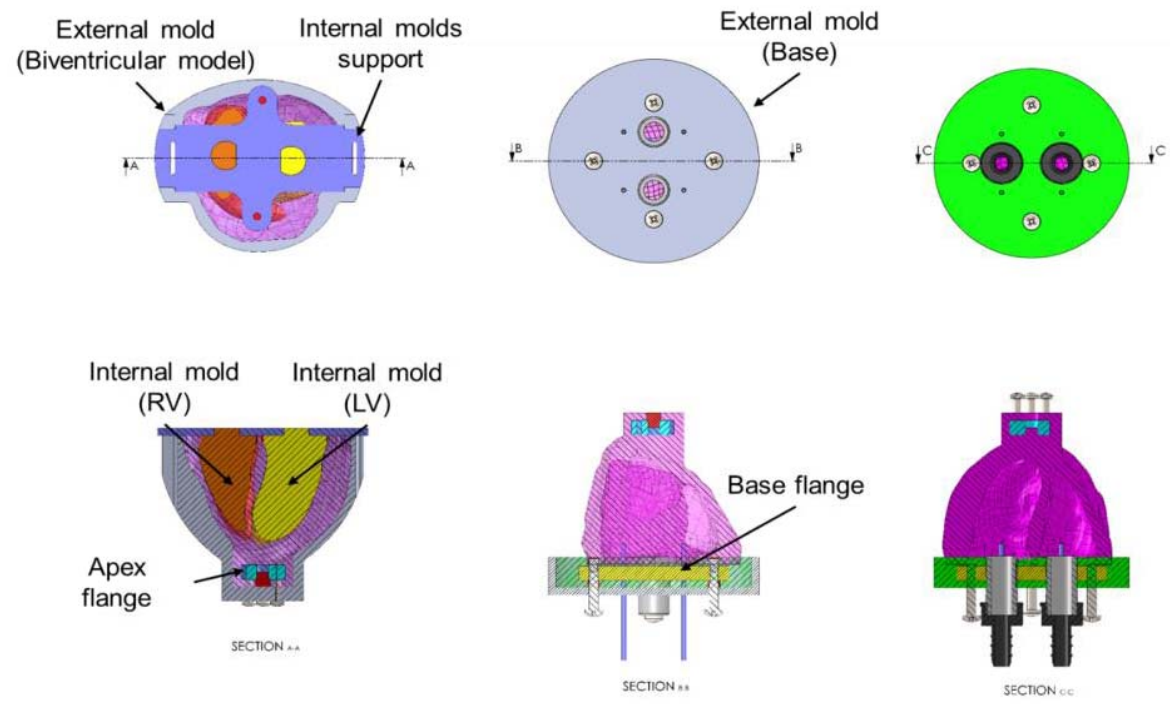

A

B

C

Fig. 5 - A - Biventricular model with apex extension. This mold is composed by the internal molds of the right ventricle (RV) and left ventricle (LV) and one external mold. Moreover, the apex flange is integrated to be encased in the phantom material. B - Base extension mold is composed by an external mold and a flange. During production, the cardiac muscle is merged to the base extension after its first freezing and thawing cycle. $\mathrm{C}-$ Final phantom model. 


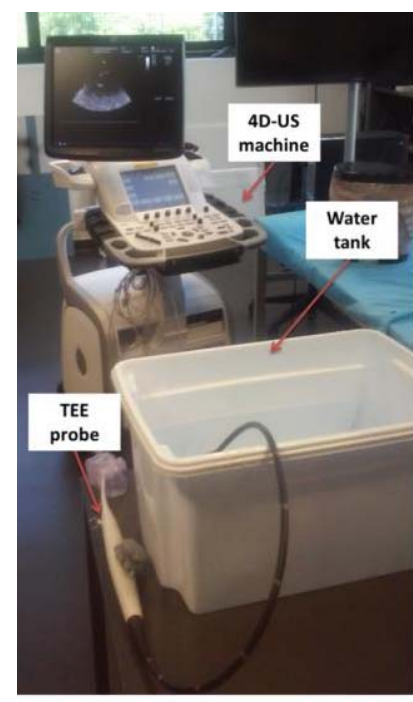

A

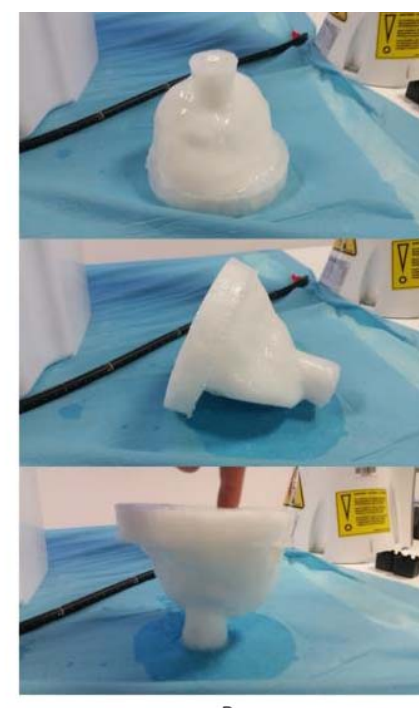

B
Fig. 6 - A - Setup for validating the acoustic properties of the biventricular phantom using a four-dimensional (4D) ultrasound (US) machine connected to a transesophageal echocardiography (TEE) probe. B

- Different viewpoints of the biventricular phantom.

by physical cross-linking through the use of freezing and thawing cycles. This technique has many advantages because it not only addresses toxicity issues but it also leads to cryogels with higher mechanical strength compared to chemical or irradiative cross-linked techniques. Thereby, the properties of the PVA-C depends on the temperature and time of freezing and thawing, as well as the molecular weight of the polymer and the concentration of the aqueous polymer solution [15]-[17].

The PVA was Mowiol 10-98 (Kuraray Europe GmbH, Hattersheim am Main, Germany) with a molecular weight of approximately 61000 and a hydrolysis degree of $98-98.8 \%$. A water solution of $15 \mathrm{wt} \%$ PVA was used. The crystals were milled and then heated to approximately $90{ }^{\circ} \mathrm{C}$ using a water bath and stirred until they became fully dissolved. Then, the solution was gradually cooled down to room temperature. The
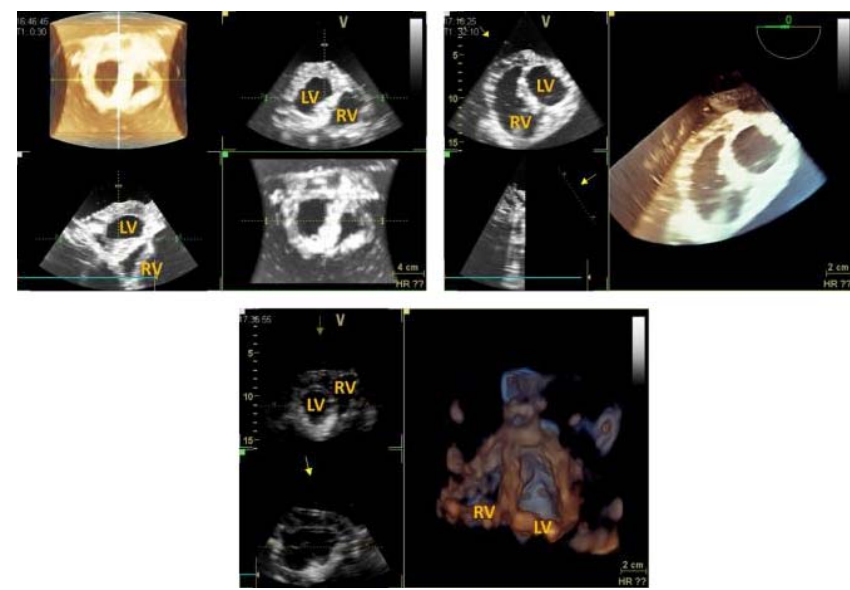

Fig. 7 - Echocardiography images of the biventricular phantom using a TEE probe. LV - Left ventricle; RV - Right ventricle. solution was poured into the mold (Fig. $1-\mathrm{F}$ ) and placed in a freezer at a temperature of $-25{ }^{\circ} \mathrm{C}$. Consecutive cycles of freezing for $24 \mathrm{~h}$ and thawing for $48 \mathrm{~h}$ were completed.

After the first cycle of freeze-thaw, the internal molds of the biventricular model were removed and the base extension was assembled. Due to this circumstance, the biventricular model performed one extra cycle (three cycles in total) compared to the base extension. After production, the PVA-C phantom was preserved in distilled water to maintain hydration.

\section{EXPERIMENTS}

Preliminary assessments were performed by inspecting, imaging, and measuring the biventricular phantom.

\section{A. Echocardiography acquisition and assessment}

An experimental setup was mounted and the cardiac phantom was submerged into a water tank to be imaged (Fig. 6-A).

A transesophageal echocardiography (TEE) probe connected to a 4D ultrasound machine (Vivid E9; GE Healthcare, Horten, Norway) was used to obtain 3D B-mode images.

The biventricular phantom was assessed by visually examining the images and measuring the volume of the LV. The commercial software available in the ultrasound machine was used to perform the LV volumetric measures, as described in Wood et al. [18].

\section{RESUlTS}

\section{A. Biventricular phantom}

The PVA-C phantom was successfully produced with a realistic texture, flexibility and resistance to deformations. Besides, the fusion between base extension and biventricular phantom, after its first freezing and thawing cycle, was achieved. Fig. 6-B presents different viewpoints of the biventricular phantom.

\section{B. Echocardiography acquisition and validation}

\section{1) Visual assessment}

The biventricular phantom was successfully imaged. Nonetheless, the walls of the water tank created echoes that hampered the image acquisitions.

The cycles of freezing and thawing gave good echogenicity to the phantom. The echocardiography images are presented in Fig. 7.

The cardiac external wall and septum presented hyperechoic characteristics and the chambers hypoechoic ones, as expected. The two chambers were clearly visible.

\section{2) Left ventricular volume evaluation}

A volume of $74 \mathrm{ml}$ (Fig. 8) was obtained using the US system, in contrast to the $98 \mathrm{ml}$ virtually designed. 


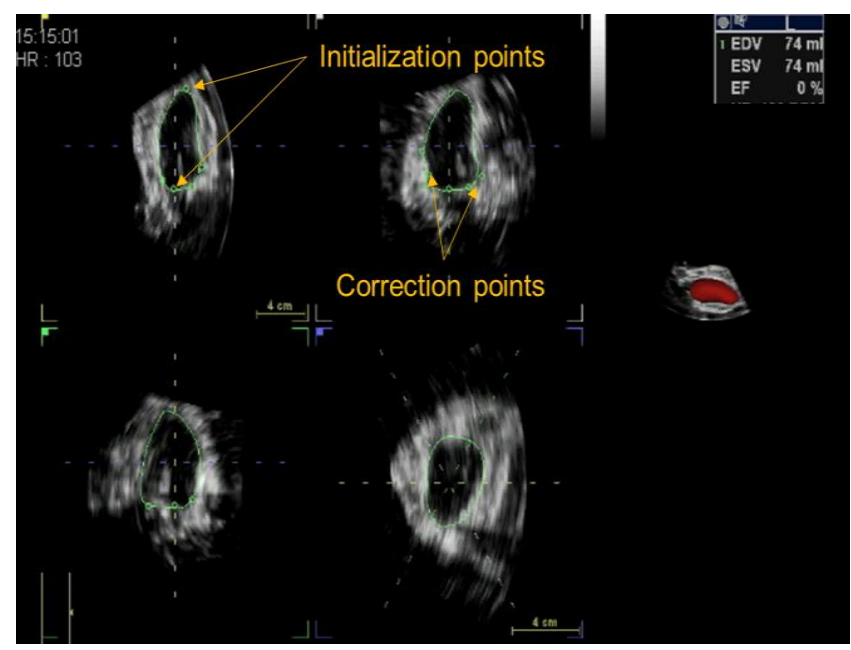

Fig. 8 - Four-dimensional automated left ventricular quantification tool (Vivid E9, GE Healthcare). Image shows different plane views of the LV. Green lines represent the automated contour computed from two initialization points. The contour can be corrected by adding points. Enddiastolic volume (EDV), end-systolic volume (ESV) and ejection fraction (EF) are computed from the delineated LV. Note that EDV and ESV were equal, with $\mathrm{EF}$ equal to $0 \%$, given that the phantom internal volume did not change during acquisition.

\section{DISCUSSION}

As described in the state-of-the-art, several studies have reported distinct approaches to develop static and/or dynamic phantoms. In this work, we developed a biventricular phantom of the heart to allow the simulation of specific cardiac interventions. The biventricular phantom is not dynamic, however it was designed to be connected to a pump and an $\mathrm{E} / \mathrm{M}$ system which will simulate the cardiac motion. The phantom was prepared to be submerged in a water tank allowing US imaging. In addition, the complete setup is tunable and uses patient-specific models. These models give the opportunity to create specific anatomies, clinical scenarios and pathologies in a controllable environment [19]. Thus, it is possible to use the same setup to study different anatomies and pathologies because the PVA-C phantom is easily replaceable. The time consumed on producing the biventricular phantom was approximately one week. The presented technique is attractive since other techniques consumed more time [10], although other less [11]. However, the time can be reduced with automatic steps, as RV and LV segmentation.

The biventricular phantom was successfully produced, replicating anatomical features of the real tissues (e.g., the echogenicity in US images). The PVA solution has a low viscosity at room temperature, which makes it versatile for filling irregularly shaped molds. In addition, the produced phantom has a low shrinkage after the freezing and thawing cycles and therefore maintain its shape after forming.

In literature, several works [3], [4], [20]-[22] report the use of $10 \mathrm{wt} \%$ aqueous solution of PVA with molecular weight 89000-98000 and hydrolysis degree >99 \% (Sigma Aldrich, St. Louis, Missouri, United States). We used $15 \mathrm{wt} \%$ aqueous solution of Mowiol 10-98 with molecular weight 61000 and hydrolysis degree of $98.0-98.8 \%$. According to
Hassan et al. [23], PVA-C with intermediate molecular weight require higher initial concentrations of aqueous PVA solutions and higher number of freezing and thawing cycles to enhance the stability during swelling in water for a long-term period, corroborating therefore the production strategy used.

As mentioned, the number of freezing and thawing cycles, PVA molecular weight and concentration of aqueous solution have a significant impact in the material properties [23], [24]. Thus, further studies must be performed to identify the optimal properties to correctly mimic the particularities of the cardiac tissue. However, this is a complex task due to the diversity of anatomical structures present in the heart (different material properties [25]) and their complex behavior (e.g., constant motion). Only by performing a complete analysis of the pressures, torsional and translational movements will be possible to understand the limitations of the current phantom. However, the main focus of this work was to develop the biventricular models to be integrated in the presented setup. As future work, the pump and the E/M system will be assembled.

The biventricular phantom revealed good echogenic properties, which made possible to visualize the anatomy of both RV and LV in the acquired echocardiographic images. The cardiac wall and septum showed high homogeneity due to the uniform material. As such, as a future work, we intend to introduce graphite in the models to enhance speckle noise in the images [3]. Moreover, an ultrasound acoustic absorber will be included in the water tank to reduce the number of echoes and improve the ultrasound acquisitions. Additionally, one expert will perform the echocardiography study in a real scenario.

The LV volume measured using the automated software was different from the virtually designed one. This may be associated with misrelated properties during US imaging, as: the water tank temperature (not $37^{\circ} \mathrm{C}$ ); different acoustic properties of the PVA-C phantom, which hampers the ultrasound measurements and LV segmentation; phantom swelling (due to continuous contact with water), which is typical in PVA-C with lower number (3 cycles) of freeze-thaw cycles [20]. As observed in [11], the PVA-C-based phantom also presented differences on volume compared to the same Silicone-based phantom and the ideal models, which suggests that it can be a typical behavior of PVA-C material.

\section{CONCLUSION}

The PVA-C phantom demonstrated to be a versatile option to produce dynamic patient-specific models of the heart ventricles. Nonetheless, several production parameters influence the PVA-C properties and its performance. Thus, extra tuning must be performed to obtain a more realistic model. Overall, the preliminary results are satisfactory and encourage the use of this methodology in the future to create phantoms to study and improve diagnosis, planning, treatment and training in the cardiovascular field.

\section{REFERENCES}

[1] R. Olszewski, Z. Trawiński, J. Wójcik, and A. Nowicki, "Mathematical and Ultrasonographic Model of the Left Ventricle: in Vitro Studies," 
Arch. Acoust., vol. 37, no. 4, pp. 583-595, Jan. 2012.

[2] B. Lesniak-Plewinska, S. Cygan, K. Kaluzynski, J. D’hooge, J. Zmigrodzki, E. Kowalik, M. Kordybach, and M. Kowalski, "A DualChamber, Thick-Walled Cardiac Phantom for Use in Cardiac Motion and Deformation Imaging by Ultrasound," Ultrasound Med. Biol., vol. 36, no. 7, pp. 1145-1156, Jul. 2010

[3] B. Heyde, S. Cygan, H. F. Choi, B. Lesniak-Plewinska, D. Barbosa, A Elen, P. Claus, D. Loeckx, K. Kaluzynski, and J. D’Hooge, "Regional cardiac motion and strain estimation in three-dimensiona echocardiography: A validation study in thick-walled univentricular phantoms," IEEE Trans. Ultrason. Ferroelectr. Freq. Control, vol. 59, no. 4, pp. 668-682, Apr. 2012.

[4] S. Cygan, K. Werys, Ł. Błaszczyk, T. Kubik, and K. Kałużyński, "Left ventricle phantom and experimental setup for MRI and echocardiography - Preliminary results of data acquisitions," Biocybern. Biomed. Eng., vol. 34, no. 1, pp. 19-24, Sep. 2014.

[5] X. Huang, J. Moore, G. Guiraudon, D. L. Jones, D. Bainbridge, J. Ren, and T. M. Peters, "Dynamic 2D ultrasound and 3D CT image registration of the beating heart," IEEE Trans. Med. Imaging, vol. 28, no. 8, pp. 1179-1189, Aug. 2009

[6] C. a Linte, J. Moore, C. Wedlake, and T. M. Peters, "Evaluation of model-enhanced ultrasound-assisted interventional guidance in a cardiac phantom.," IEEE Trans. Biomed. Eng., vol. 57, no. 9, pp. 2209 2218, Sep. 2010.

[7] X. Huang, J. Ren, G. Guiraudon, D. Boughner, and T. M. Peters, "Rapid dynamic image registration of the beating heart for diagnosis and surgical navigation.," IEEE Trans. Med. Imaging, vol. 28, no. 11, pp. 1802-1814, Nov. 2009

[8] C. Vannelli, J. Moore, J. McLeod, D. Ceh, and T. Peters, "Dynamic heart phantom with functional mitral and aortic valves," in Proc. SPIE 9415, Medical Imaging 2015: Image-Guided Procedures, Robotic Interventions, and Modeling, 2015, vol. 9415, p. 941503:1-10.

[9] M. E. Rettmann, D. R. Holmes, D. M. Kwartowitz, M. Gunawan, S. B. Johnson, J. J. Camp, B. M. Cameron, C. Dalegrave, M. W. Kolasa, D. L. Packer, and R. A. Robb, "Quantitative modeling of the accuracy in registering preoperative patient-specific anatomic models into left atrial cardiac ablation procedures," Med. Phys., vol. 41, no. 2, p. 21909, Jan. 2014.

[10] J. Laing, J. Moore, D. Bainbridge, M. Drangova, and T. Peters, "Patient-specific atrium models for training and pre-procedure surgical planning,” 2017, vol. 10135, p. 101351A.

[11] P. Morais, J. M. R. S. Tavares, S. Queirós, F. Veloso, J. D’hooge, and J. L. Vilaça, "Development of a patient-specific atrial phantom model for planning and training of inter-atrial interventions," Med. Phys., vol. 44, no. 11, pp. 5638-5649, Nov. 2017.

[12] OsiriX, "DICOM sample image sets." [Online]. Available: http://www.osirix-viewer.com/datasets/.

[13] D. Maleike, M. Nolden, H.-P. Meinzer, and I. Wolf, "Interactive segmentation framework of the Medical Imaging Interaction Toolkit," Comput. Methods Programs Biomed., vol. 96, no. 1, pp. 72-83, Oct. 2009.

[14] A. Fetzer, S. Zelzer, T. Schroeder, H.-P. Meinzer, and M. Nolden, "An interactive 3D segmentation for the Medical Imaging Interaction Toolkit (MITK)," in Proc. MICCAI Interactive Medical Image Computing, 2014, pp. 1-11.

[15] K. J. M. Surry, H. J. B. Austin, A. Fenster, and T. M. Peters, "Poly(vinyl alcohol) cryogel phantoms for use in ultrasound and MR imaging.," Phys. Med. Biol., vol. 49, no. 24, pp. 5529-5546, Dec. 2004.

[16] C. M. Hassan and N. a Peppas, "Structure and Applications of Poly(vinyl alcohol) Hydrogels Produced by Conventional Crosslinking or by Freezing/Thawing Methods," in Biopolymers · PVA Hydrogels, Anionic Polymerisation Nanocomposites, vol. 153, Berlin, Heidelberg: Springer Berlin Heidelberg, 2000, pp. 37-65.

[17] S. R. Stauffer and N. a. Peppast, "Poly(vinyl alcohol) hydrogels prepared by freezing-thawing cyclic processing," Polymer (Guildf)., vol. 33, no. 18, pp. 3932-3936, Sep. 1992.

[18] P. W. Wood, P. H. Gibson, and H. Becher, "Three-dimensional echocardiography in a dynamic heart phantom: comparison of five different methods to measure chamber volume using a commercially available software," Echo Res. Pract., vol. 1, no. 2, pp. 51-60, Oct. 2014.

[19] S. N. Kurenov, C. Ionita, D. Sammons, and T. L. Demmy, "Three- dimensional printing to facilitate anatomic study, device development, simulation, and planning in thoracic surgery," J. Thorac. Cardiovasc. Surg., vol. 149, no. 4, p. 973-979.e1, Apr. 2015

[20] V. Tavakoli, M. Kendrick, M. Shakeri, M. Alshaher, M. F. Stoddard, and A. Amini, "A multimodal (MRI/ultrasound) cardiac phantom for imaging experiments," in Proc. SPIE 8672, Medical Imaging 2013. Biomedical Applications in Molecular, Structural, and Functional Imaging, 2013, vol. 8672, p. 867202:1-8

[21] J. A. Minton, A. Iravani, and A.-M. Yousefi, "Improving the homogeneity of tissue-mimicking cryogel phantoms for medical imaging.," Med. Phys., vol. 39, no. 11, pp. 6796-6807, Nov. 2012.

[22] V. Tavakoli, M. J. Negahdar, M. Kendrick, M. Alshaher, M. Stoddard, and A. A. Amini, "A biventricular multimodal (MRI/ultrasound) cardiac phantom," in 34th Annual International Conference of the IEEE Engineering in Medicine and Biology Society, 2012, pp. 3187-3190.

[23] C. M. Hassan and N. A. Peppas, "Structure and Morphology of Freeze/Thawed PVA Hydrogels," Macromolecules, vol. 33, no. 7, pp. 2472-2479, Apr. 2000

[24] S. Cournane, L. Cannon, J. E. Browne, and A. J. Fagan, “Assessment of the accuracy of an ultrasound elastography liver scanning system using a PVA-cryogel phantom with optimal acoustic and mechanical properties," Phys. Med. Biol., vol. 55, no. 19, pp. 5965-5983, Oct. 2010.

[25] H. Wen, E. Bennett, N. Epstein, and J. Plehn, "Magnetic resonance imaging assessment of myocardial elastic modulus and viscosity using displacement imaging and phase-contrast velocity mapping," Magn. Reson. Med., vol. 54, no. 3, pp. 538-548, Sep. 2005. 